(C2019, Elsevier. Licensed under the Creative Commons Attribution-NonCommercialNoDerivatives 4.0 International http://creativecommons.org/about/downloads

cc) 


\section{The opinion of different observer groups about the aesthetic impact and need for surgical correction of varying submental lengths}

\section{Abstract}

Purpose: This study evaluated the opinion of different observer groups about the influence of submental length on perceived attractiveness and when surgical correction was deemed necessary.

Methods: The submental length of an idealized silhouette of a male Caucasian profile was altered incrementally between 5 and $95 \mathrm{~mm}$. Images were rated for attractiveness on a Likert scale by pretreatment orthognathic surgery patients $(n=75)$, lay persons $(n=75)$, and clinicians (maxillofacial surgeons and orthodontists) $(\mathrm{n}=35)$.

Results: For perceived attractiveness, the ideal submental length was approximately $50 \mathrm{~mm}$ (range: 40 to $75 \mathrm{~mm}$ ). A submental length shorter than or equal to $30 \mathrm{~mm}$ was deemed unattractive by all 3 groups. Overall, a submental length less than $40 \mathrm{~mm}$ generally was judged to be less attractive than a comparable increase in length. Clinicians were generally least likely to suggest surgery for varying submental lengths. For this group, the cut-off where the majority suggested surgery was a submental length of $25 \mathrm{~mm}$ or less. For the patient and lay groups, the corresponding cut-off values were a length shorter than or equal to $30 \mathrm{~mm}$ or equal to $95 \mathrm{~mm}$. Conclusions: A submental length of approximately $50 \mathrm{~mm}$ (range 40 to $75 \mathrm{~mm}$ ) was viewed by most observers as attractive. From $30 \mathrm{~mm}$ and less, it was generally deemed progressively less attractive. Clinicians were less likely to suggest corrective surgery than the patient and lay groups. For comparative proportional relationships, submental length should be between the lower lip-chin height and lower facial height, assuming an otherwise proportional facial profile.

Keywords: Submental length, throat length, chin-neck aesthetics, profile aesthetics 


\section{Introduction}

There are two facial profile parameters that are fundamental to the perception of submental-cervical aesthetics. The first is the submental-cervical angle, which has received considerable attention in the clinical literature, ${ }^{1-11}$ and the second is submental length, which is potentially equally as important, but has received comparatively limited consideration in the literature.

Submental length, sometimes referred to as "throat length", may be defined as the horizontal distance from C-point to a vertical tangent through soft tissue pogonion when the patient's head is in natural position. C-point is defined as the innermost (posterior-superior) point between the submental plane and the anterior aspect of the neck in the midsagittal plane, located at the intersection of lines drawn tangential to the submental region and the anterior neck (Figure 1).

The submental length and submental-cervical angle may be altered with soft tissue surgical procedures, for example by platysmal plication or submental liposuction, or by surgical advancement or set-back of the mandible and/or osseous chin with orthognathic surgery. ${ }^{12}$ The submental length is a clinically relevant parameter in treatment planning for mandibular set-back or set-back osseous genioplasty, as both procedures effectively reduce submental length. Therefore, in presurgical patients with average or reduced submental length, the surgeon may tend toward greater maxillary advancement as opposed to mandibular setback, or at least consider limiting the bony movements in order to avoid an unattractively short submental length postoperatively.

For any given population, the submental length will have an 'average' value or 'norm' specific for age, gender and ethnicity, and a range of normal variability, with appearance concerns often resulting from a significant deviation of the submental length proportion from the accepted norm for the population. For clinical diagnosis and treatment planning, it is 
important to know at what point the submental length proportion moves from the acceptable range of variability to being perceived as a facial deformity.

The magnitude of the deviation, whether it is due to the underlying mandibular-chin skeletal framework, the submental soft tissue thickness or a combination of the two, is an important factor in deciding when orthognathic surgery and/or submental soft tissue surgery may be required. If the magnitude of the discrepancy of submental length is great, the treatment planning decision may be relatively straightforward. However, a significant number of patients are regarded as "borderline" in terms of need for treatment. In such patients, the decisionmaking process may be transferred from subjective clinical judgement to objective, evidencebased guidance based on the data from studies investigating perceptions of submental length and attractiveness.

The principal aim of this investigation was to evaluate quantitatively the influence of

submental length and its proportion on perceived facial attractiveness. The relationship between submental length and attractiveness was recorded to ascertain the range of normal variability in terms of observer acceptance, and to determine the clinically significant threshold value or cut-off point beyond which the length is perceived as unattractive and surgical correction is desired. The perceptions of pre-orthognathic surgery patients, clinicians (maxillofacial surgeons, orthodontists), and lay persons were compared for these different variables.

\section{Materials and methods}

Ethical approval was obtained from the National Research Ethics Service (NRES), United Kingdom (REC reference: 06/Q0806/46).

A profile silhouette image was created with computer software (Adobe Photoshop CS2 software, San Jose, CA). Such two-dimensional profile silhouettes are used routinely to assess the perceptions of facial attractiveness. ${ }^{13,14}$ The image was manipulated using the same 
software to construct an "ideal" profile image with proportions ${ }^{15}$ and linear and angular softtissue measurements ${ }^{6,8,15,16}$ based on currently accepted criteria for an idealized Caucasian male profile, as previously described. ${ }^{14}$ The submental length of the idealized image (image BA: $50 \mathrm{~mm}$ ) was then altered in $5-\mathrm{mm}$ increments from $5 \mathrm{~mm}$ to $95 \mathrm{~mm}$ to create a range of images representing variations of submental length from reduced to potentially excessive (Figure 2). Based on the results of a pilot study and power calculation, 185 observers took part in the study, separated into three groups (75 pretreatment orthognathic surgery patients, 75 lay persons, and 35 clinicians (Table 1), with the following selection criteria:

1. Patients: pretreatment (having had only one consultation appointment); primary concern was facial appearance; no previous facial surgical treatment; no history of facial trauma; no severe psychological issues (based on patient interview by the lead investigator).

2. Lay persons: no previous facial surgery, deformities, or history of facial trauma.

3. Clinicians: involved in the management of patients with facial deformities; included 19 maxillofacial surgeons and 16 orthodontists with 1 to 16 years of experience in the clinical management of patients requiring orthognathic and facial reconstructive surgery.

Each observer was asked to rate each image in terms of facial attractiveness using the following rating scale:

1. Extremely unattractive

2. Very unattractive

3. Slightly unattractive

4. Neither attractive nor unattractive

5. Slightly attractive 


\section{Very attractive \\ 7. Extremely attractive}

Observers were also asked whether they would consider surgery to correct the submental length if this was their facial appearance (yes or no).

The images were placed in random order into the software application Microsoft PowerPoint (Microsoft Corporation, Redmond, WA). Each image was identified by a randomly assigned double letter in the top right corner of the screen (e.g., BA, CB etc.). One duplicate image was included to assess intra-examiner reliability (images DD and EA). Each observer sat undisturbed in the same room in front of the same computer and 17-in. flat screen monitor. The images were created in such a way that each of the profile silhouette images when viewed on the monitor had the same dimensions as a normal human head, reducing the potential effect of image size on observer perception. Each observer examined the images in the PowerPoint presentation by pressing the "Page Down" button on the keyboard at their own pace. The sevenpoint Likert scale was used by each observer to rate each image in terms of attractiveness. The Likert-type rating scale is largely accepted in the psychology literature as the most useful rating method. ${ }^{17}$

\section{Statistical analysis}

Descriptive statistics such as median and interquartile ranges of the attractiveness ratings in each observer group were calculated for individual submental lengths with software that we developed using MATLAB (The MathWorks Inc., Natick, MA). Similarly, the software calculated the proportion in each group suggesting a need for surgery. Additional data analysis and graphs were prepared using Excel (Microsoft Corporation, USA). 


\section{Results}

\section{Reliability Analysis}

For the patients and lay groups there was a one-point difference in the median attractiveness score for the identical images (DD and EA) indicating reasonable repeatability; for the clinician group the median attractiveness score was the same for the two identical images with a submental length of $40 \mathrm{~mm}$ (Figure 3).

Table 2 shows the interquartile range of Likert scores; the median interquartile range was two for lay and patient groups and one for the clinician group indicating that there was generally reasonable agreement among each group. For one of the two identical images in the patient group with a submental length of $40 \mathrm{~mm}$, there was an interquartile range of three, whereas for the clinician group the maximum interquartile range was two.

\section{Perceived attractiveness of images}

Figure 3 shows the median attractiveness rating of the observers on a Likert scale from 1 to 7 , where 1 indicates 'extremely unattractive' and 7 indicates 'extremely attractive'; overall there were similar median rankings between the groups for most submental lengths. Table 3 shows the data from Figure 3 in rank order from most to least attractive, sorted based on responses from the clinician group. A submental length less than or equal to $30 \mathrm{~mm}$, or equal to $95 \mathrm{~mm}$, was associated with a reduction in the median attractiveness scores to below 4 in the lay and clinician groups; for the patient group the corresponding range was less than or equal to $30 \mathrm{~mm}$ or greater than or equal to 85 to $95 \mathrm{~mm}$ (Figure 3).

\section{Most attractive and least attractive images}

Table 4 indicates the proportion of observers suggesting surgery who considered attractiveness to be important ( $>2$ on a scale of 1 to 4 ). For patients it was $68 / 75$, and all the clinicians considered attractiveness to be important; overall there was generally reasonable 
agreement particularly among patients and lay persons as to whether surgery is required with a slightly lower proportion of clinicians suggesting surgery. Figure 4 shows the proportion of clinicians suggesting surgery compared with submental length. Thus, taking $50 \%$ as a level for suggesting surgery, the lay and clinician groups considered this to apply for a submental length less than or equal to $25 \mathrm{~mm}$ and for the patient group the corresponding length was less than or equal to $30 \mathrm{~mm}$ or equal to $95 \mathrm{~mm}$.

When assessing the proportion of each observer group suggesting surgery is required, the clinicians were generally least likely to suggest surgery for the varying submental lengths. Repeatability of the 35 clinicians' assessments appears reasonable, with no clinicians suggesting surgery for one of the two identical images. For the 75 lay persons the assessment of the two repeated images was for one $11 \%$ and the other $37 \%$ suggesting surgery and there was also some difference for the group of 75 patients, with proportions of $13 \%$ and $29 \%$.

\section{Discussion}

Submental length and its proportional relationship to the facial soft tissue profile is a significant facial aesthetic parameter. In terms of perceived attractiveness, the results of this investigation demonstrate that the ideal submental length appears to be approximately $50 \mathrm{~mm}$, with a range of between 40 to $75 \mathrm{~mm}$ depending on the observer group. A submental length shorter than or equal to $30 \mathrm{~mm}$ was deemed unattractive by all 3 groups. In the lay and clinician groups, a submental length equal to $95 \mathrm{~mm}$ was associated with a reduction in attractiveness ratings, and for the patient group the corresponding range was greater than or equal to 85 to 95 $\mathrm{mm}$. A reduction in submental length below $40 \mathrm{~mm}$ appeared generally to be judged as being more unattractive than a comparable increase in length.

Overall, the perceptions of the three observer groups in this investigation were relatively similar, albeit with the pretreatment orthognathic surgery patient group being very slightly more critical of the appearance of the images. It can be speculated that living with a 
perceived dentofacial deformity makes patients more aware of the facial appearance of others and this can lead to their developing a greater sensitivity to noticeable differences in facial appearance from the 'ideal' compared to lay or clinician groups.

In terms of the need for surgical correction, it is of interest that the results indicate that the clinicians were generally less likely than the other two groups to suggest surgery for varying submental lengths. This may be a result of their greater clinical experience with such patients and a more realistic understanding of the burden of such treatment on patients, having previously witnessed them going through these operations.

As with any facial parameter, it is generally acknowledged that the submental length has a range of normal individual variability. As a starting point for comparative purposes and to assess potential similarities and differences, it is worthwhile to look at the submental length and its proportional relationships in idealized images from classical and Renaissance art and sculpture (Table 5). The first known treatise on ideal human proportions, known as the Canon, was written by the Greek sculptor Polykleitos of Argos. ${ }^{15}$ Unfortunately, no copies of this book exist. However, it is known, based on evidence from the physician Galen, that Polykleitos based his most important statue, the Doryphoros, on this treatise. The submental length proportion in these statues is approximately equal to the lower lip and chin height in both males and females (Table 5). During the Italian Renaissance, which preceded the Renaissance in the rest of Europe by about a century, a number of famous artists and sculptors attempted to define ideal facial proportions in order to improve their works. Leonardo da Vinci described "ideal" submental length as equal to the distance between the mouth (stomion) and the bottom of the chin (menton), which is a quarter of facial height. ${ }^{18,19}$ Leonardo based his proportional relationships on anthropometric measurements of attractive individuals, as did Leon Battista Alberti before him. ${ }^{15,20}$ In some of his paintings, e.g. La Bella Principessa, the submental length is slightly shorter than lower lip-chin height (Table 5). However, for a number of other 
Renaissance artists, such as Albrecht Dürer, the most famous German Renaissance artist, Titian and Botticelli, their very well-known images of "ideal" beauty portray the submental length as longer than lower lip-chin height, and in fact equal to the lower anterior facial height (subnasale to menton) (Table 5). From a number of idealized male and female profile images painted or sculpted, whether in classical Greece or Rome, or in the Renaissance, the submental length proportion appears to be either close to the lower lip-chin height or lower anterior facial height. The common denominator in the morphology of the submental region in these images is that it is not shorter than lower lip-chin height to any appreciable degree (Table 5).

Although Farkas ${ }^{8,16}$ provided average values for many facial parameters based on anthropometric studies on North American adults of white ethnicity, Chinese-Americans and African-Americans, unfortunately he did not anthropometrically measure the submental length in any of his investigations. However, Farkas $^{8}$ did provide the following values for lower lipchin height (stomion-menton), which were $50 \pm 4 \mathrm{~mm}$ in white males and $45 \pm 3 \mathrm{~mm}$ in white females, and the lower lip-chin height was proportionally close to the submental length according to a number of the classical and Renaissance artists mentioned above. Legan and Burstone $^{7}$ described the proportional relationship between lower facial height and submental length (measured from C-point to a tangent to pogonion) as 1:2, i.e. for the same lower face height, a ratio greater than 1:2 relates to a reduced submental length. The relevance of these anthropometric values is due to the comparative proportional relationship of the submental length as being approximately between the lower lip-chin height and the lower facial height, assuming an otherwise proportional facial profile, as described above.

Additionally, a number of modern surgical authorities have provided "ideal" values for the submental length, based either on anecdotal evidence and the "good eye" of the respective surgeon or on data from their treated cases. Some authorities measured submental length as the linear distance from C-point to soft tissue menton with the patient's head in natural position, 
and others as the linear distance from C-point to a tangent to soft tissue pogonion (Figure 1), which would be a somewhat longer distance than the former. For example, Worms et al. ${ }^{9}$ provided a normative value of $57 \pm 6 \mathrm{~mm}$ measured from C-point to menton, whereas Moreno et al. ${ }^{10}$ provided a value of $62 \pm 6 \mathrm{~mm}$ measured from C-point to a pogonion tangent. Epker and Stella ${ }^{1}$ provided a value of $50 \mathrm{~mm}$ measured from C-point to a pogonion tangent, and Sommerville et al. ${ }^{11}$ used a slightly different approach and provided a value of $51 \mathrm{~mm}$ (range $32 \mathrm{~mm}-73 \mathrm{~mm}$ ), measured from C-point to a tangent to a line joining subnasale and pogonion.

The results of this investigation have clinical treatment implications. For example, the submental length tends to be reduced in patients with true mandibular deficiency and increased in those with true mandibular excess. A mandibular advancement or osseous advancement genioplasty will tend to increase the submental length, which is usually an aesthetic improvement. Conversely, a mandibular set-back or set-back osseous genioplasty will reduce the submental length and may lead to increased 'submental fullness,' which is undesirable. Therefore, submental length and its proportional relationships should be considered in planning orthognathic surgery. For example, in a patient with a Class III skeletal relationship, mandibular set-back procedures may need to be avoided or at least limited if the submental length is average or reduced, with the planning clinician opting for a proportional greater maxillary advancement rather than mandibular set-back.

It is important to bear in mind that the profile silhouette images created in this study were based on North American white adult male proportions and normative values. As such, it may not be generalizable to different ethnic groups, although it does provide some insight into how different ethnic groups view faces of white ethnicity. It would be interesting to repeat the study using images from different ethnic groups. It should also be specified that the results may not apply directly to white females, which is another area that may be explored in future investigations. It is worth noting that the proportion of female clinicians acting as observers in 
this investigation was higher than that represented in the oral and maxillofacial surgery profession, and the mean age was relatively young. It is also worth mentioning that twodimensional profile silhouettes do not provide a true representation of the relative midface deficiency that presents as mandibular length increases in real life three-dimensional perspective. The implications of the two latter points on the results are unclear but may be worthy of further exploration in future studies.

\section{Conclusions}

A submental length of approximately $50 \mathrm{~mm}$, with a range of 40 to $75 \mathrm{~mm}$, appears to be viewed by most observers in this study as attractive. From $30 \mathrm{~mm}$ and less, submental length is generally deemed progressively less attractive. Reduced submental length, between 5 and 35 $\mathrm{mm}$, appears to be less attractive than increased submental length in the range 35 to $90 \mathrm{~mm}$. In terms of need for surgery, clinicians and the lay group suggested surgery at a submental length less than or equal to $25 \mathrm{~mm}$ and the patient group for a submental length less than or equal to $30 \mathrm{~mm}$ or equal to $95 \mathrm{~mm}$. A comparative proportional relationship that may be useful is that the submental length should be approximately between the lower lip-chin height and the lower facial height, assuming an otherwise proportional facial profile. 


\section{REFERENCES}

1. Epker, B.N., Stella, J.P. Systematic aesthetic evaluation of the neck for cosmetic surgery. Oral Maxillofac Surg Clin North Am. 2:217, 1990

2. Rees, T.D., Wood-Smith, D. Cosmetic Facial Surgery. WB Saunders Company, Philadelphia; 1973

3. Skoog, T. Plastic Surgery: New Methods and Refinements. WB Saunders Company, Philadelphia; 1974

4. Guyuron, B., Jackowe, D., Iamphongsai, S. Basket submandibular gland suspension. Plast Reconstr Surg. 122:938, 2008

5. Ellenbogen, R., Karlin, J.V. Visual criteria for success in restoring the youthful neck. Plast Reconstr Surg. 66:826, 1980

6. Powell, N., Humphreys, B. Proportions of the Aesthetic Face. Thieme-Stratton Inc., New York; 1984

7. Legan, H., Burstone, C.J. Soft tissue cephalometric analysis for orthognathic surgery. J Oral Surg. 38:744; 1980

8. Farkas, L.G. (Ed.). Anthropometry of the Head and Face. $2^{\text {nd }}$ Ed.. Raven Press, New York; 1994

9. Worms, F.W., Isaacson, R.J., Speidel, T.M. Surgical orthodontic treatment planning: profile analysis and mandibular surgery. Angle Orthod. 46:1, 1976

10. Moreno, A., Bell, W.H., You, Z.H. Esthetic contour analysis of the submental cervical region: a study based on ideal subjects and surgical patients. $J$ Oral Maxillofac Surg. 52:704, 1994

11. Sommerville, J.M., Sperry, T.P., BeGole, E.A. Morphology of the submental and neck region. Int J adult Orthod Orthognathic Surg. 3:97, 1988 
12. Gill, D.S., Lloyd, T., East, C., Naini, F.B. The facial soft tissue effects of orthognathic surgery. Facial Plast Surg. 33:519, 2017

13. Ioi, H., Nakata, S., Nakasima, A., Counts, A. Effect of facial convexity on anteroposterior lip positions of the most favored Japanese facial profiles. Angle Orthod. $75: 326,2005$

14. Naini, F.B., Donaldson, A.N.A., McDonald, F., et al. Assessing the influence of chin prominence on perceived attractiveness in the orthognathic patient, layperson and clinician. Int J Oral Maxillofac Surg. 41:839, 2012

15. Naini, F.B. Facial Aesthetics: Concepts and Clinical Diagnosis. Oxford, WileyBlackwell, Oxford, UK; 2011

16. Farkas, L.G., Kolar, J.C., Munro, I.R. Geography of the nose: a morphometric study. Aesthetic Plast Surg. 10:191, 1986

17. Langlois, J.H., Kalakanis, L., Rubenstein, A.J., et al. Maxims or myths of beauty? A meta-analytic and theoretical review. Psychol Bull. 126:390, 2000

18. Richter, J.P. Literary Works of Leonardo da Vinci. $2^{\text {nd }}$ Ed. Oxford University Press, Oxford, UK; 1939

19. Kemp, M. (Ed.). Trans. Kemp, M., Walker, W. Leonardo on Painting. Yale University Press, New Haven; 1989

20. Naini, F.B., Cobourne, M.T., Garagiola, U., et al. Nasofacial angle and nasal prominence: A quantitative investigation of idealized and normative values. $J$ Craniomaxillofac Surg. 44:446, 2016 


\section{Table captions}

Table $1 \quad$ Observer demographics.

Table 2 Interquartile range of attractiveness rankings of the Likert score. The two identical images (DD and EA) are shown in italics.

Table 3 Data in rank order from most to least attractive sorted by clinician ranking. The two identical images (DD and EA) are shown in italics.

Table 4 Proportion of observers suggesting surgery who considered attractiveness to be important. The two identical images (DD and EA) are shown in italics.

Table 5 The proportion of submental length to lower lip and chin height in idealized images from classical and Renaissance art and sculpture.

\section{Figure captions}

Figure 1 C-point (cervical point), defined as the innermost point between the submental region and the anterior surface of the neck in the midsagittal plane.

Sn (subnasale), defined as the deepest midline point where the base of the nasal columella meets the upper lip;

Pog' (soft tissue pogonion), defined as the most prominent midline point of the soft tissue chin pad;

Me' (soft tissue menton), defined as the most inferior midline point of the soft tissue chin pad;

Sti (stomion inferius), the most superior midline point of the lower lip.

Submental length, linear distance from C-point to a vertical tangent through Pog' (although some authorities measure it from C-point to Me').

Lower lip and chin height, linear distance from Sti to a horizontal tangent through Me'.

Lower anterior face height (LAFH), linear distance from Sn to a horizontal tangent through $\mathrm{Me}^{\prime}$. 
Figure 2 The submental length of the idealized image was altered incrementally to create a range of images. The two-letter code assigned to each image and the submental length in millimetres shown in this figure are provided on each image for clarity; for data collection the submental length in millimetres was not on the images shown to observers, and the two-letter code was located outside each image on the computer monitor background.

Figure 3 Median attractiveness rating against submental length for patients (dashed and dotted line), lay people (dashed line) and clinicians (dotted line). The median attractiveness differs at most by one between the groups and for nine of the nineteen submental length values the median rating is the same for all three groups.

Figure 4 Proportion of clinicians suggesting surgery based on submental length. 